TITLE:

\title{
Rise of iPSCs as a cell source for adoptive immunotherapy.
}

AUTHOR(S):

Minagawa, Atsutaka; Kaneko, Shin

CITATION:

Minagawa, Atsutaka ...[et al]. Rise of iPSCs as a cell source for adoptive immunotherapy.. Human cell 2014, 27(2): 47-50

ISSUE DATE:

2014-04

URL:

http://hdl.handle.net/2433/199830

\section{RIGHT:}

The final publication is available at Springer via http://dx.doi.org/10.1007/s13577-0140089-8.; This is not the published version. Please cite only the published version.; この論 文は出版社版でありません。引用の際には出版社版をご確認ご利用ください。 


\title{
Rise of iPSCs as a cell source for adoptive immunotherapy
}

\author{
Atsutaka Minagawa, M.D. and *Shin Kaneko, M.D., Ph.D. \\ Shin Kaneko laboratory, Department of Cell Growth and Differentiation, Center \\ of iPS cell Research and Application (CiRA), Kyoto University. \\ *correspond to kaneko.shin@cira.kyoto-u.ac.jp
}

\begin{abstract}
Adoptive T-cell transfer is a potentially effective strategy for treating cancer and viral infections. However, previous studies of cancer immunotherapy have shown that $\mathrm{T}$ cells expanded in vitro fall into an exhausted state and, consequently, have limited therapeutic effect. One way to overcome this obstacle is to use induced pluripotent stem cells (iPSCs) as a cell source for making effector T cells. In recent years, there have been several reports on generating effector $\mathrm{T}$ cells suitable for adoptive immunotherapy. The reported findings suggest that by using iPSC technology, it may be possible to stably derive large numbers of juvenile memory $\mathrm{T}$ cells targeted to cancers or viruses. In this review, we describe a strategy for applying iPSC technology to immunotherapy and the characteristics of $\mathrm{T}$ cells derived from iPSCs. We also discuss how these
\end{abstract}


technologies can be applied clinically in the future.

\section{Introduction}

$\mathrm{T}$ cells play a central role in acquired immunity against pathogens. Since the identification of tumor-specific antigens and their epitopes in the 1990's (1), substantial progress has been made in our understanding of $\mathrm{T}$ cell-mediated antitumor responses. From that understanding emerged adoptive cell transfer (ACT) therapy for cancer, which makes use of tumor-infiltrating lymphocytes (TILs) and has achieved some success. For example, Rosenberg's group in National Cancer Institute reported that after lymphodepleting systemic chemotherapy, adoptive transfer of TILs induced clear and reproducible responses in nearly $50 \%$ of melanoma patients (2). Despite the therapeutic potential of TIL infusion therapy against cancer and chronic viral infections, there remain two important biological problems must be overcome (3-5). One is that cancer and viruses often hamper or escape $\mathrm{T}$ cell immunity by increasing regulatory $\mathrm{T}$ cell (Treg) counts, expressing one our more inhibitory molecules (e.g., PD-1L), or suppressing antigen presentation. This can be partially remedied by removing/remodeling the host immune system using chemoradiotherapy or newly developed monoclonal antibodies that have immunoregulatory effects $(2,6)$. The other 
problem relates to the quality of $\mathrm{T}$ cells expanded ex vivo. As currently performed, in vitro expansion for ACT induces $\mathrm{T}$ cells to differentiate into a late effector state and increase killer activity; however these cells then fall into an exhausted state and do not survive or proliferate in vivo after infusion. Consequently, they have little therapeutic effect $(2,7)$. Optimization of the in vitro culture protocol may improve outcomes, but for now the problem remains to establish antigen-specific $\mathrm{T}$ cell clones that need repeated stimulation in long-term culture periods.

To overcome that problem, the therapeutic potential of induced pluripotent cells (iPSCs) is drawing attention (8). iPSCs have the capacity for self-renewal while maintaining pluripotency and could potentially be a major cell source for induction of juvenile $\mathrm{T}$ cells suitable for ACT therapy. $\mathrm{T}$ cells recognize antigens via their $\mathrm{T}$ cell receptors (TCRs). Because TCR genes are irreversibly rearranged in the thymus during $\mathrm{T}$ cell maturation, iPSCs derived from $\mathrm{T}$ cells (T-iPSCs) retain the rearranged TCR genes of the original cell. It would therefore be expected that redifferentiating T-iPSCs derived from antigen-specific $\mathrm{T}$ cells would produce large numbers of juvenile antigen-specific $\mathrm{T}$ cells. In mouse, the therapeutic potential of this approach was successfully demonstrated through nuclear transplantation and subsequent in vivo maturation using toxoplasma gondii antigen-specific $\mathrm{T}$ cells and alpha-GalCel-specific 
Valpha14 NKT cell nuclei $(9,10)$.

Generation of iPSCs from T cells was initially difficult. But over the years several groups, including ours, have succeeded in using integrated viral vector systems to produce T-iPSCs from mouse and human polyclonal and antigen-specific T cells (11-16). This has enabled the potential of T-iPSCs as a cell source for ACT to be explored all around the world.

\section{Generation of rejuvenated antigen-specific $\mathbf{T}$ cells using T-iPSCs}

In 2013, a series of studies were reported on the establishment of T-iPSCs from antigen-specific cytotoxic $\mathrm{T}$ cells (CTLs) and redifferentiation of the T-iPSCs into functional CTLs $(16,17)$. Two Japanese groups, including ours, each succeeded in using a Sendai viral vector to establish T-iPSCs, our group from a HIV nef protein-specific CTL clone and Kawamoto's group from a melanoma MART-1 antigen-specific CTL clone. To differentiate T-iPSCs into CTLs, we employed an in vitro differentiation protocol entailing co-culture with C3H10T1/2 and Delta-like 1-expressing OP9(OP9-DL1) stromal cells. After 35-40 days of differentiation, we obtained CD3+ CD4+ CD8+ double-positive (DP) stage T cells. It is at this stage during physiological thymocyte development that TCR- $\alpha$ gene rearrangement occurs $(18,19)$. With 
T-iPSC-derived DP cells, activation of Rag-1 and Rag-2, two genes related to recombination machinery, was observed, as was a reduction in the percentage of original antigen-specific cells. To create mature CD8 single-positive (SP) CTLs from T-iPSCs without TCR gene revision, it was necessary to artificially end the TCR- $\alpha$ rearrangement. TCR signaling via the peptide-major histocompatibility complex (MHC) during positive selection is known to downregulate RAG expression and prevent further rearrangement of TCR (19). In addition, stimulation using a anti-CD3 antibody is known to mimic TCR signals (20). Both our group and Kawamoto's stimulated differentiated T-iPSCs on days 35-40 using anti-CD3 antibody or PHA and succeeded in producing CD8 SP cells. In our hands, redifferentiated CD8 SP cells recognized the same peptide on HIV antigen as the original CTLs, and immunological assays revealed that these cells exhibit normal cytolytic activity, INF- $\gamma$ secretion and degranulation when stimulated by their target peptide. In addition, proliferation assays revealed that redifferentiated T-iPSCs had greater expansion potential and longer telomeres than the original cells, indicating CTLs can become "rejuvenated" by passing through the T-iPSC state.

Clonal expansion of acquired immune cells is very useful for clinical application, as has been seen with B cell monoclonal antibodies. This strategy to generate $\mathrm{T}$ cells by 
passing them through T-iPSCs enables us to expand clinically applicable $\mathrm{T}$ cell clones

stably and without limit, which could drive innovation in ACT therapy.

Decades of research into cancer immunology have established that strong lymphocyte infiltration is associated with a good outcome in many types of tumors, including melanoma and head and neck, breast, renal, bladder, urotherial, ovarian, colorectal and pancreatic cancers, among others. This provides hope that ACT could become an effective therapy for numerous tumor types (21). At present, however, clinical trials of ACT are performed only in a few tumors, like melanoma. This is in part because of the difficulty of achieving proliferation of tumor-specific CTLs, which must differ radically depending on the immunogenicity of the tumor (22). A stable and unlimited supply of tumor-specific CTLs derived from T-iPSCs will facilitate research into ACT therapy for many types of cancer not treatable with immunotherapy at present.

\section{iPSC based expansion of Mucosal-associated invariant T}

In $\mathrm{T}$ cells there are some subsets of innate $\mathrm{T}$ cells as represented by natural killer $\mathrm{T}$ (NKT) cells other than acquired immune $\mathrm{T}$ cells. Mucosal associated invariant $\mathrm{T}$ (MAIT) cells belong to the innate $\mathrm{T}$ cells and are abundant in humans, representing up to $50 \%$ of the resident $\mathrm{T}$ cells in the Liver, $10 \%$ of the peripheral blood mononuclear 
cells (PBMCs) were known to play a pivotal role in host defense against a wide range of bacterial and fungal infection including mycobacterium. Recently, Wakao et al reported generating T-iPSCs from human cord blood MAIT cells by using Sev vector and redifferentiated them into MAIT cells. Redifferentiated MAIT (re-MAIT) cells could produce various cytokines such as IFN- $\gamma, \mathrm{TNF}-\alpha$ in the presence of bacteria-fed monocyte as original MAIT cells do. Infusion of re-MAIT cells into immunocompromised nice showed their antimycobacterial activity in vivo (23).

This result not only demonstrate the clinical potential of T-iPSCs derived innate T cells, but also show the possibility of T-iPSC technology as a device to explore the rare or difficult to culture $\mathrm{T}$ cell populations

\section{Combination of T-iPSC and CAR technology}

Genetic engineering of $\mathrm{T}$ cells to express chimeric antigen receptors (CARs) has recently emerged as a promising approach to rapidly generating tumor-targeted $\mathrm{T}$ cells endowed with enhanced antitumor properties (24). Most CARs utilize an antigen-derived antigen-binding motif to recognize their target (25). This enables them to recognize cell surface antigens with a higher affinity than TCRs and to function in an HLA-independent fashion, thereby eliminating the need to consider HLA restriction and 
overcoming some tumor escape mechanisms. Recently clinical trials of CD19

CAR-modified $\mathrm{T}$ cells demonstrated their efficient targeting of acute and chronic lymphoblastic leukemias (26-30). Moreover, promising results on the use T-iPSCs as a cell source for CAR-modified T cells were recently reported (31). By itself, use of CAR technology is a new and potentially problematic strategy, and severe adverse effects have been reported in several clinical trials. But although many aspects of this approach require improvement, the combination of iPSC and CAR technologies offers a promising avenue to the treatment of cancers.

\section{Discussion}

T-iPSCs possess great potential as a cell source for ACT therapy. Establishing T-iPSCs from a patient's own antigen specific T lymphocytes and redifferentiating them for ACT therapy is an ideal way to tailor immunotherapy. But this strategy still has significant problems, including the time required and cost. For example, it takes at least 3 months to establish T-iPSCs and redifferentiate them into T cells, and establishing iPSCs for each patient may be too costly for routine use. One possible solution is to generate a T-iPSC bank for common combination of cancer antigen and HLA haplotypes (i.e HLA-A2 restricted cancer antigen WT1 derived specific peptide) and repress 
allo-antigenic HLA expression through genomic modification $(32,33)$. If such an antigen-HLA-identified T-iPSC bank could be established, then the dream of coordinating antigen-specific CTLs for timely ACT therapy based on T-iPSCs with matched HLA-type might come true. This banking concept would match well with CAR or exogenous TCR transduction technology to T-iPSC.

Increasing the efficiency of T-iPSC generation and redifferentiation, establishment of feeder-free culture systems for $\mathrm{T}$ cells, cost reduction, and many biological and technical challenges lie ahead. But if it were possible to achieve T-iPSCs as a cell source for ACT therapy, then the treatment of malignancies and chronic viral infections would be completely changed, and the impact on medicine would be enormous. 


\section{Reference}

1. van der Bruggan $\mathrm{P}$, Traversari $\mathrm{C}$, Chomez $\mathrm{P}$, et al. A gene encoding an antigen recognized by cytolytic $\mathrm{T}$ lymphocytes on a human melanoma. Science 254:1643-1647,1991

2. Dudley ME, Yang JC, Sherry R, et al. Adoptive cell therapy for patients with metastatic melanoma: evaluation of intensive myeloablative chemoradiation preparative regimens. J Clin Oncol 26:5233-5239, 2008

3. Gattinoni L. Adoptive immunotherapy for cancer: building on success. Nat Rev immunol 6:383-393, 2006

4. Campoli M, Ferrone S. HLA antigen changes in malignant cells: epigenetic mechanisms and biologic significance. Oncogene 27:5869-5885, 2008

5. Viguier M, Lemaitre F, Verola O, et al. Foxp3 expressing CD4+CD25(high) regulatory $\mathrm{T}$ cells are overrepresented in human metastatic melanoma lymph nodes and inhibit the function of infiltrating T cells. J immunol 173:1444-1453, 2004

6. Devaud C, John LB, Westwood JA, et al. Immune modulation of the tumor microenvironment for enhancing cancer immunotherapy. Oncoimmunology 2:e25961, 2013

7. Gattinoni L, Klevanoff CA, Palmer DC, et al. Acquisition of full effector function in vitro paradoxically impairs the in vivo antitumor efficacy of adoptively transferred CD8+ T cells. J Clin Invest 115:1616-1626, 2005

8. Takahashi K, Tanabe K, Ohnuki M, et al. Induction of pluripotent stem cells from adult human fibroblasts by defined factors. Cell 131: 2007, 861-72

9. Kirak O, Frickel EM, Grotenbreg GM, et al. Transnuclear mice with predefined T cell receptor specificities against Toxoplasma gondii obtained via SCNT. Science 328:243-248, 2010

10. Watari H, Fujii S, Yamada D, et al. Murine induced pluripotent stem cells can be derived from and differentiate into natural killer T cells. J Clin invest 120:2610-2618, 2010

11. Brown ME, Rondon E, Rajesh D, et al. Derivation of induced pluripotent stem cells from human peripheral blood T lymphocytes. Plos One 5:e11373, 2010

12. Seki T, Yuasa S, Oda M, et al. Generation of induced pluripotent stem cells from human terminally differentiated circulating T cells. Cell Stem Cell 7:11-14 2010

13. Loh $\mathrm{YH}$, Hartung $\mathrm{O}, \mathrm{Li} \mathrm{H}$, et al. Reprogramming of $\mathrm{T}$ cells from human peripheral blood. Cell Stem Cell 7:15-19, 2010

14. Staerk J, Dawlaty MM, Gao Q, et al. Reprogramming of human peripheral blood cells to induced pluripotent stem cells. Cell Stem Cell 7:20-24, 2010 
15. Hong H, Takahashi K, Ichisaka T, et al. Suppression of induced pluripotent stem cell generation by the p53-p21 pathway. Nature 460:1132-5, 2009

16. Nishimura T, Kaneko S, Kawana AT, et al. Generation of rejuvenated antigen-specific $\mathrm{T}$ cells by reprogramming to pluripotency and redifferentiation. Cell Stem Cell 12:114-126, 2013

17. Vizcardo R, Masuda K, Yamada D, et al. Regeneration of human tumor antigen-specific T cells from iPSCs derived from mature CD8(+) T cells. Cell Stem Cell 12:31-36, 2013

18. von Boehmer H. Selection of the T-cell repertoire: receptor-controlled checkpoints in T-cell development. Adv immunol 84:201-238, 2004

19. Krangel MS. Mechanics of T cell receptor gene rearrangement. Curr Opin immunol 21:133-139, 2009

20. Turka LA, Schatz DG, Oettinger MA, et al. Thymocyte expression of RAG-1 and RAG-2: termination by T cell receptor cross-linking. Science 253:778-781, 1991

21. June $\mathrm{CH}$. Adoptive $\mathrm{T}$ cell therapy for cancer in the clinic. $\mathrm{J}$ Clin Invest 117:1466-1476, 2007

22. Cheever MA, Allison JP, Ferris AS, et al. The prioritization of cancer antigens: a national cancer institute pilot project for the acceleration of translational research. Clin Cancer Res 15:5323-5337, 2009

23. Wakao H, Yoshikiyo K, Koshimizu U, et al. Expansion of functional human mucosal-associated invariant $\mathrm{T}$ cells via reprogramming to pluripotency and redifferentiation. Cell Stem Cell 12:546-558, 2013

24. Sadelain M, Brentjens R, Riviere I. The promise and potential pitfalls of chimeric antigen receptors. Curr Opin Immunol 21:215-223, 2009

25. Sadelain M, Brentjens R, Riviere I. The basic principles of chimeric antigen receptor design. Cancer Discov 3:388-398, 2013

26. Kalos M, Levine BL, Porter D, et al. T cells with chimeric antigen receptors have potent antitumor effects and can establish memory in patients with advanced leukemia. Sci Transl Med 3:95ra73, 2011

27. Kochenderfer JN, Dudley ME, Caarpenter RO, et al. Donor-derived CD19-targeted $\mathrm{T}$ cells cause regression of malignancy persisting after allogeneic hematopoietic stem cell transplantation. Blood 122:4129-4139, 2013

28. Till BG, Jensen MC, Wang J, et al. CD20-specific adoptive immunotherapy for lymphoma using a chimeric antigen receptor with both CD28 and 4-1BB domains: pilot clinical trial results. Blood 119:3940-3950, 2012

29. Porter DL, Levine BL, Kalos M, et al. Chimeric antigen receptor-modified T cells in chronic lymphoid leukemia. NEJM 365:725-733, 2011 
30. Grupp SA, Kalos M, Barret D, et al. Chimeric antigen receptor-modified T cells for acute lymphoid leukemia. NEJM 368:1509-1518, 2013

31. Themeli M, Kloss CC, Ciriello G, et al. Generation of tumor-targeted human T lymphocytes from induced pluripotent stem cells for cancer therapy. Nat Biotechnol 31:928-933, 2013

32. Riolobos L, Hirata RK, Turtle CJ, et al. HLA engineering of human pluripotent stem cells. Mol Ther 21:1232-1241, 2013

33. Haruta M, Tomita Y, Yuno A, et al. TAP-deficient human iPS cell-derived myeloid cell lines as unlimited cell source for dendritic cell-like antigen-presenting cells. Gene Ther 20:504-513, 2013 